\title{
Gender insensitive salutations in letters and correspondence sections of journals
}

\author{
Satish Suhas ${ }^{1}\left[\right.$ Prabha S. Chandra ${ }^{1} \cdot$ Santosh K. Chaturvedi ${ }^{1}$ \\ Received: 20 August 2021 / Accepted: 15 October 2021 / Published online: 27 October 2021 \\ (c) The Author(s), under exclusive licence to Springer-Verlag GmbH Austria, part of Springer Nature 2021
}

Globally there is a significant transformation in the knowledge and attitudes with an increasing emphasis on gender-inclusive language and behavior. Although gender-inclusive language is emphasized in scientific publishing, much progress is needed to overcome the archaic stereotypes. Unfortunately, there is still a gender imbalance among the chief editors of major scientific journals, including psychiatry (Roberts 2014; Hafeez et al. 2019). However, despite this, we think it would be better to use gender-neutral language in salutations used in correspondence sections of scientific publications. Although much more effort is needed to overcome other gender-related issues in research, gender-inclusive scientific culture can address seemingly minor issues, such as genderinsensitive salutations in scientific manuscripts.

1. Salutation in published correspondence: Several psychiatry journals continue to publish correspondence articles or letters to the editor, starting with the salutation as "Sir." Authors must be aware that the gender or the gender preference of the editor may be different from the one assumed from the information available in the public domain. Additionally, the continued use of this archaic salutation promotes gender stereotypes and does not befit gender neutrality. There are hardly any journals that begin a correspondence article starting with "Madam." It is therefore recommended that all correspondence and letters to editors begin with "Dear Editor" instead.

2. Addressing editors in covering letters: Covering letters is often the author's first impression in the editor's eyes. It must

Satish Suhas

suhasedu@yahoo.in

Prabha S. Chandra

prabhasch@gmail.com

Santosh K. Chaturvedi

skchatur@gmail.com

1 Department of Psychiatry, National Institute of Mental Health and Neurosciences, Bangalore 560 029, India be framed to prevent gender stereotypy and cisgenderism in covering letters addressed to editors. Scientific journals may provide templates with explicit instructions on formats of the covering letter to carry salutation as "To the Editor" rather than "Dear Sir" or "Dear Madam."

3. Salutation preference in editorial submission software: Leading journals continue to use salutations based on binary gender dichotomy. Gender transcendence can be achieved through the insistence of asking authors about their gender preference salutation rather than a dropdown menu of options based on binary gender identity salutation. This will prevent inadvertent stigma and promotes self-preference in gender narratives.

Although there are complex gender neutrality issues that need to be addressed in scientific literature, perhaps it can start with a change in salutations used. The scientific publication ethos in psychiatry can provide the impetus towards this need to unlearn and relearn to overcome archaic salutations and promote gender neutrality in scientific communications.

Author contribution Dr Satish Suhas, Dr Santosh Kumar Chaturvedi, and Dr Prabha S Chandra conceptualized the idea and prepared the manuscript.

\section{Declarations}

Conflict of interest The authors declare no competing interests.

\section{References}

Hafeez DM, Waqas A, Majeed S et al (2019) Gender distribution in psychiatry journals' editorial boards worldwide. Compr Psychiatry 94:152119. https://doi.org/10.1016/j.comppsych.2019.152119 Roberts LW (2014) Where are the women editors? Acad Psychiatry 38:391-393. https://doi.org/10.1007/s40596-014-0173-0

Publisher's note Springer Nature remains neutral with regard to jurisdictional claims in published maps and institutional affiliations. 